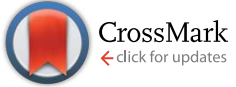

Cite this: Soft Matter, 2015, 11, 972

\title{
Enzymatically crosslinked dendritic polyglycerol nanogels for encapsulation of catalytically active proteins $\uparrow$
}

\begin{abstract}
Changzhu Wu, ${ }^{a}$ Christoph Böttcher ${ }^{\mathrm{b}}$ and Rainer Haag *a
The enormous potential of nanogel scaffolds for protein encapsulation has been widely recognized. However, constructing stable polymeric nanoscale networks in a facile, mild, and controllable fashion still remains a technical challenge. Here, we present a novel nanogel formation strategy using horseradish peroxidase (HRP) catalyzed crosslinking on phenolic derivatized dendritic polyglycerol (dPG) in the presence of $\mathrm{H}_{2} \mathrm{O}_{2}$ in an inverse miniemulsion. This "enzymatic nanogelation" approach was efficient to produce stable $200 \mathrm{~nm}$ dPG nanogel particles, and was performed under physiological conditions, thus making it particularly beneficial for encapsulating biological proteins. Purification of the nanogels was easy to handle and practical because there was no need for a post-quenching step. Interestingly, the use of dPG resulted in higher HRP laden nanogels than for linear polyethylene glycol (PEG) analogs, which illustrates the benefits of dendritic backbones in nanogels for protein encapsulation. In addition, the mild immobilization contributed to the enhanced thermal stability and reusability of HRP. The nanogel preparation could be easily optimized to achieve the best HRP activity. Furthermore, a second enzyme, Candida antarctica lipase B (CalB), was successfully encapsulated and optimized for activity in dPG nanogels by the same enzymatic methodology, which shows the perspective applications of such techniques for encapsulation of diverse proteins.
\end{abstract}

Received 6th August 2014

Accepted 11th December 2014

DOI: $10.1039 / c 4 s m 01746 c$

www.rsc.org/softmatter temperature and extreme $\mathrm{pH}$, which compromises their functions during biological or industrial use. Alternatively nanogels can also be produced by a template-assisted chemical crosslinking of hydrophilic polymers or monomers. ${ }^{1,6}$ The advantages of the chemical approach are the covalently connected networks which enable nanogels stably transported during biomedical applications or withstand extreme industrial application conditions. The used templates can be a heterogeneous colloidal environment, such as an inverse miniemulsion where chemical crosslinking is restricted to the volume of aqueous nano-droplets, ${ }^{11-13}$ or it can be a defined mold to shape the network formation in nanoscale. ${ }^{14}$ However, the main concern in many chemical approaches is the presence of residual, cytotoxic crosslinking agents or an unavoidable exposure to harsh experimental conditions. Therefore, most physical and chemical approaches are not suitable for the preparation of stable nanogels under physiological conditions.

Unlike traditional physical and chemical methods, biological enzymes can mediate polymer crosslinking with high selectivity and specificity under physiologic conditions, which avoids unwanted side products and minimizes the damage of surrounding bioactive agents. ${ }^{15}$ As a result, enzymatic crosslinking for in situ bulk hydrogel formation is becoming a rapidly growing area of research in the fields of biomaterials, ${ }^{16,17}$ tissue engineering, ${ }^{15,18}$ drug delivery, ${ }^{19}$ and regenerative medicine. ${ }^{20}$ Despite these obvious advantages, enzymatic crosslinking for 
nanogel preparation has been relatively under-explored, although it has a high potential for significantly promoting the development of biomacromolecule loaded nanogels. The only example reported to date was from Groll and co-workers who formulated nanogels with redox-sensitive disulfide networks by horseradish peroxidase (HRP) in the absence of hydrogen peroxide. ${ }^{3}$ This system has proven to be particularly effective for encapsulating active $\beta$-galactosidase. But apart from this approach, the combined use of $\mathrm{HRP}$ and $\mathrm{H}_{2} \mathrm{O}_{2}\left(\mathrm{HRP} / \mathrm{H}_{2} \mathrm{O}_{2}\right)$ has been widely used to form bulk hydrogel because of its faster and in particular more controllable gelation process. ${ }^{15,16,18-21}$ Although the presence of $\mathrm{H}_{2} \mathrm{O}_{2}$ has the potential to create a cytotoxic environment for cells or to deactivate proteins, the rate of $\mathrm{H}_{2} \mathrm{O}_{2}$ consumption can be explicitly controlled by manipulating its initial concentration for crosslinking. ${ }^{16} \mathrm{Up}$ to now, however, such efficient $\mathrm{HRP} / \mathrm{H}_{2} \mathrm{O}_{2}$ system has never been exploited for nanogel fabrication.

Besides the difficulty in finding suitable crosslinking conditions, another big obstacle that prohibits practical applications of nanogels is the requirement for a tedious nanogel separation. For example, nanogel preparation in a classic inverse emulsion requires multiple washing and dialysis steps to transfer particles from the organic phase into water., ${ }^{3,11-13}$ In particular, post-quenching residual crosslinkable groups on the nanogel surface is essential for avoiding further nanogel crosslinking in the separation steps, which turns out to be expensive and is toxic for the encapsulated bioactive units. Therefore, there has been a quest to establish a reliable and effective methodology to facilitate nanogel purification for biomedical or industrial use.

Herein we describe a new nanogel preparation method for protein encapsulation by HRP-catalyzed oxidative crosslinking on 3-(4-hydroxyphenyl) propionic acid functionalized dendritic polyglycerol (dPG-HPA) in the presence of $\mathrm{H}_{2} \mathrm{O}_{2}$ in an inverse miniemulsion (Scheme 1). Compared with traditional inverse emulsion systems for the preparation of linear polymer network nanogels, the current method has four distinct advantages: (1)

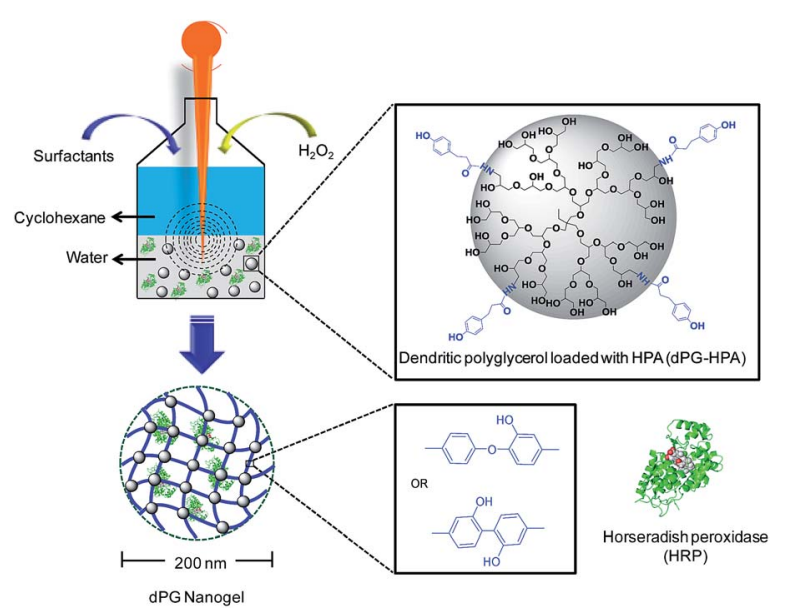

Scheme 1 Schematic representation of dPG nanogel preparation via $\mathrm{HRP}$ crosslinking on dPG-HPA in the presence of $\mathrm{H}_{2} \mathrm{O}_{2}$ in an inverse miniemulsion. mild crosslinking conditions, (2) a facile nanogel preparation without post-quenching, (3) efficient protein loading derived from the closely-packed dendritic backbone of networks, and (4) multiple ways to optimize enzyme activity in scaffolds because of controllable gelation process and multi-functionality of dPG scaffolds. To the best of our knowledge, this is the first example of a nanogel fabrication combining $\mathrm{HRP}$ and $\mathrm{H}_{2} \mathrm{O}_{2}$ in a controllable fashion. Moreover, we demonstrate dPG nanogels provide more efficient scaffolds for active protein encapsulation for biocatalysis compared to linear analogs.

\section{Results and discussion}

\section{dPG nanogel fabrication}

The HRP-catalyzed oxidative crosslinking procedure was used to generate nanogels in a water-in-oil inverse miniemulsion template stabilized by the bioinert surfactants Span 80 and Tween 80 (Scheme 1). Hydrogen peroxide $\left(\mathrm{H}_{2} \mathrm{O}_{2}\right)$ was used to react with HRP to form highly reactive intermediates for crosslinking of polymers that contain phenolic moieties. ${ }^{22}$ In order to minimize the residual $\mathrm{H}_{2} \mathrm{O}_{2}$ content in nanogels to facilitate separation and reduce harmful effects, an excessive amount of dPG-HPA and a sufficient reaction time were applied for the crosslinking (Experimental section). After 2 hours, no residual $\mathrm{H}_{2} \mathrm{O}_{2}$ could be detected by quantitative peroxide assay (see the ESI $\dagger$ ), which indicates that nearly all the $\mathrm{H}_{2} \mathrm{O}_{2}$ was consumed in the aqueous phase, making further crosslinking of nanogels impossible. Based on this fact, transferring nanogels from the continuous organic phase into water was directly conducted by stepwise washing out organic solvents and surfactants by cyclohexane, methanol, and water, respectively. In comparison to traditional emulsion polymerization approaches, the current method does not require any postquenching step to terminate remaining functional groups before nanogel separation, which greatly facilitates the preparation process, avoids further use of toxic reagents, and is definitely beneficial for bio-related nanogel applications.

The nanogels were characterized by dynamic light scattering (DLS) and cryogenic transmission electron microscopy (cryoTEM), respectively. The DLS data in Fig. 1a shows that no crosslinking took place in the absence of either $\mathrm{H}_{2} \mathrm{O}_{2}$ or HRP for two hours and only polymeric precursors or enzymes with diameters smaller than $10 \mathrm{~nm}$ were present in water after removal of the organic solvents. In contrast, the enzymatic crosslinking was observed in the presence of both HRP and $\mathrm{H}_{2} \mathrm{O}_{2}$, resulting in the formation of water-soluble nanogel particles with diameters of approximately $195 \pm 5 \mathrm{~nm}$ and PDI $0.075 \pm 0.04$. The formed nanoparticles were of perfect spherical geometry and an average diameter of around $200 \mathrm{~nm}$ was further confirmed by cryo-TEM image data (Fig. 1b, ESI $\dagger$ ). Stereo cryo-TEM image pairs (Fig. S17, ESI†) confirmed spherical shape and porous network architecture of nanogel particles which are particularly beneficial for a diffusive uptake of substrates in the context of biomedical applications. The obtained nanogels via $\mathrm{HRP} / \mathrm{H}_{2} \mathrm{O}_{2}$ crosslinking have much narrower PDI and smaller size comparing to those ones formed with HRP in the absence of $\mathrm{H}_{2} \mathrm{O}_{2}$ by Groll et al. ${ }^{3}$ This 


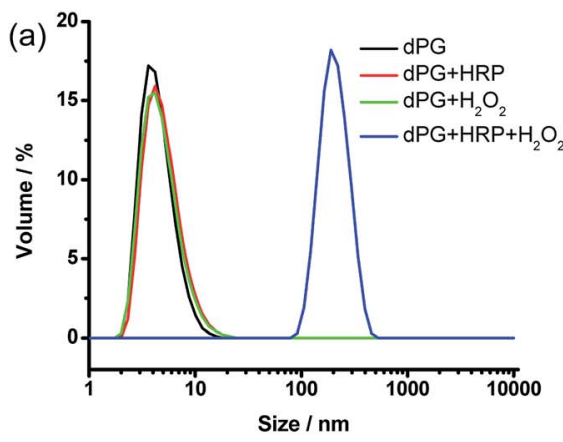

(b)

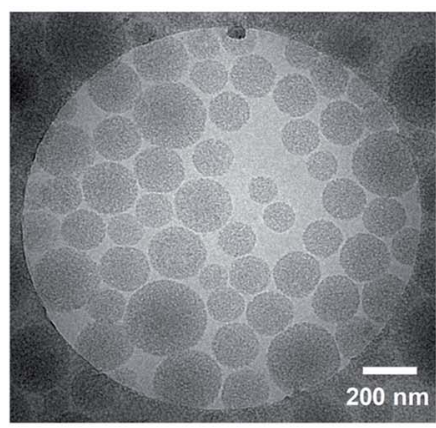

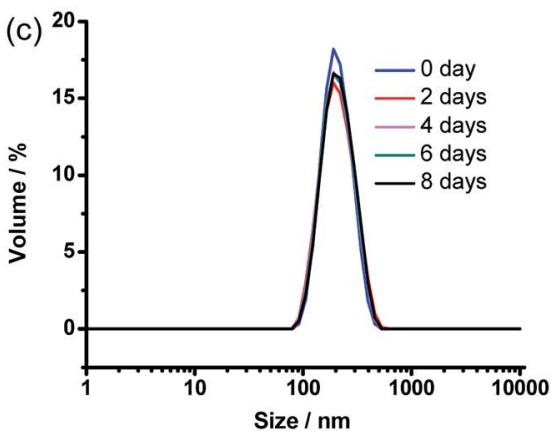

Fig. 1 Nanogel characterization: (a) DLS data of dPG nanogel formation with or without $\mathrm{H}_{2} \mathrm{O}_{2}$ and HRP, (b) cryo-TEM images of dPG nanogels, and (c) DLS data of dPG nanogel stability in water. The nanogels were typically formed by $100 \mathrm{mg} \mathrm{mL}^{-1} \mathrm{dPG}^{-2 \% \mathrm{HPA}, 0.25 \mathrm{mg} \mathrm{mL}}{ }^{-1} \mathrm{HRP}$, and $14 \mathrm{mM} \mathrm{H}_{2} \mathrm{O}_{2}$.

improvement should be attributed to a more controllable gel formation and facile separation of the current method. Moreover, dPG nanogels were found to be stable in water for over a week without any size change (shown in Fig. 1c), which reflects their strong gel networks and makes them suitable carriers for durable encapsulation of biomacromolecules.

\section{Release, thermal stability, and reuse of HRP-laden nanogels}

Recent studies disclosed the distinct protein leaching profile between nanogels and bulk hydrogels as a result of different mesh size. ${ }^{3,23,24}$ In order to explore the difference, dPG bulk hydrogels and nanogels were prepared with the same concentration of $\mathrm{HRP}, \mathrm{H}_{2} \mathrm{O}_{2}$ and dPG-2\% HPA and protein release was monitored during the process of gel preparation as well as their further incubation in water. The HRP release profile was evaluated by determining the released HRP activity from an incubation solution of nano-/hydrogels via a chromogenic reaction using ABTS as substrates (Experimental section). Fig. 2a show that up to $85 \%$ HRP could be initially encapsulated in dPG bulk hydrogels immediately after gel formation. But these enzymes were quickly released from bulk hydrogel scaffolds when further incubated with water for 72 hours. The complete leaching of HRP was attributed to the larger mesh size of hydrogel networks than HRP, which is in line with our recently revealed rheological data. ${ }^{21}$ Distinct from bulk gels, only $40 \%$ proteins were finally loaded into nanogels after being transferred into water. The reduced encapsulation efficiency in nanogels is thought to be protein loss during nanogel purification, as well as to protein interactions/adsorptions of surfactants that were used for creating miniemulsion. ${ }^{25}$ Surprisingly, as long as nanogels were transferred into water, there was no further observed protein release for 72 hour incubation (Fig. 2a), eventually resulting in $1.64 \mu \mathrm{g}_{\text {protein }}$ per $\mathrm{mg}_{\text {nanogels }}$ protein loading in nanogels. One reason for the negligible protein leaching in dPG nanogels could be that the smaller network mesh size was formed in nanogels than bulk hydrogels as already suggested by Groll et al..$^{3}$ Another possibility might be due to the globular architecture of dPG backbones which could be connected as closely-packed scaffolds. In order to verify this hypothesis, tyramine conjugated linear polyethylene glycol (PEG-TG, see the synthesis in the ESI $\dagger$ ) was enzymatically formulated into nanogels under the same conditions as $\mathrm{dPG}-2 \%$ HPA nanogels. Even though linear PEG had the same molecule weight and number of phenol groups as $\mathrm{dPG}-2 \% \mathrm{HPA}$, the initial HRP encapsulation was four times lower in PEG nanogels than dPG nanogels. Further observation revealed that encapsulated HRP were continuously released from PEG nanocarriers over 72 hours as result of the severe protein leaching profile of linear PEG nanogels. All together, enzymatic crosslinking in dendritic polymers formed more compact nanogel networks,
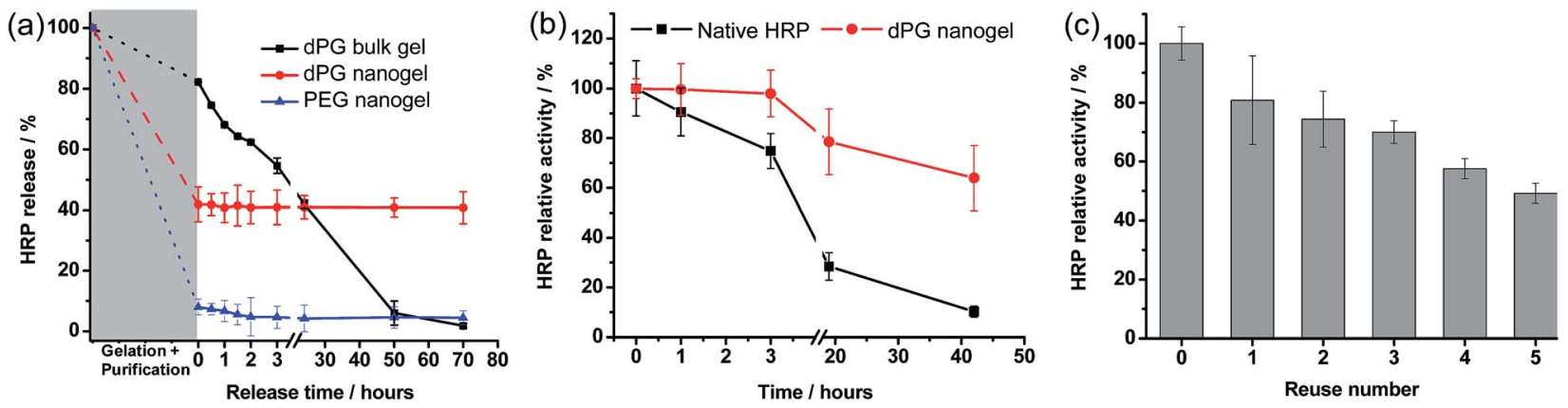

Fig. 2 The performance of encapsulated HRP: (a) HRP release profile of dPG nano- and hydrogels, and PEG nanogels, (b) thermal stability of native HRP and dPG nanogels, and (c) dPG nanogel reuse. The nanogels were formulated by $100 \mathrm{mg} \mathrm{mL}^{-1} \mathrm{dPG}^{-2 \% ~} \mathrm{HPA}, 0.25 \mathrm{mg} \mathrm{mL}{ }^{-1} \mathrm{HRP}$, and $14 \mathrm{mM} \mathrm{H}_{2} \mathrm{O}_{2}$. 
which contributed to a more efficient and stable HRP encapsulation.

Enzymes entrapped in a matrix behave significantly different from their native states because of the distinct surrounding microenvironment. In order to examine protein thermal stability, both native and encapsulated HRP were subjected to $50{ }^{\circ} \mathrm{C}$ incubation for 42 hours. Fig. $2 \mathrm{~b}$ shows that encapsulated HRP was quite stable at such high temperature, and nearly $70 \%$ HRP activity was eventually retained after incubation. In contrast, native HRP quickly deactivated over 42 hours with an overall $90 \%$ activity loss. The calculated data (see the ESI $\dagger$ ) further disclosed that the half-life time $\left(t_{1 / 2}\right)$ of encapsulated HRP was 5.6 times enhanced compared to the native HRP. The prolongation of the thermal stability points to the high potential of HRP encapsulated nanogels as practical biocatalysts for asymmetric synthetic transformations, where high operation temperatures are sometimes beneficial.

Industrial applications necessitate not only a high thermal stability of enzymes, but also their multiple usability, which is particularly essential for cost-effective production. The native HRP could hardly be reused because of the difficulty to isolate small proteins with only $\sim 4 \mathrm{~nm}$ diameters from reaction media. ${ }^{3,26}$ Data from Fig. 1b and 2a indicate that HRP could be easily entrapped into $200 \mathrm{~nm}$ dPG nanogels through "selfcatalyzed" crosslinking, which eventually equipped them as biocatalytically active nanoparticles with facilitated reuse. The obtained nanogels could be reused up to 5 times and still maintained approximately $50 \%$ activity of HRP, as shown in Fig. 2c. Therefore, encapsulating HRP in dPG nanogels makes the biocatalysts reusable, which will reduce synthetic cost and facilitate the separation process for applications.

\section{Optimization of HRP activity in dPG nanogels}

Although there are advantages with respect to thermal stability and reuse of immobilized HRP, the preparation of nanogels has to be optimized in order to achieve an optimal formulation for enzyme encapsulation. For the optimization, a conversion of chromogenic substrate of ABTS by HRP was chosen to determine enzyme activity (U) (Experimental section), and enzyme stability was studied by incubation of HRP in different preparation conditions, and expressed by the change of enzyme relative activity (\%). The obtained nanogels were concentrated into one $\mathrm{mL}$, and their total catalytic performance was quantified by the volumetric activity $\left(\mathrm{U} \mathrm{mL}^{-1}\right)$.

We first optimized nanogel yield because higher yield is known to benefit protein encapsulation.$^{27}$ In order to achieve an optimal nanogel yield, crosslinking was conducted with dPG loaded with 2, 4, 6, and $8 \%$ phenol groups, respectively. More than $8 \%$ phenol loading resulted in the decreased water-solubility of dPG, which made a further increase the degree of substitution (DS) of polymers undesirable for nanogel preparation. ${ }^{1} \mathrm{H}$ NMR spectroscopy was employed to quantify the degree of HPA substitution to polymers, which is calculated by integrating the aromatic peaks at $\delta 7.09$ and $6.80 \mathrm{ppm}$ as well as by the dPG scaffold peaks at $\delta 3.37-4.10$ ppm, as shown in Fig. 3 .

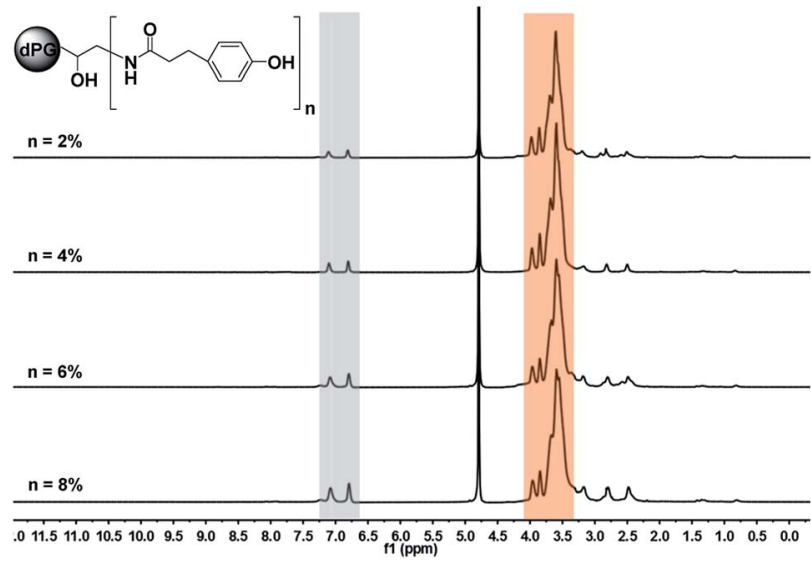

Fig. $3{ }^{1} \mathrm{H}$ NMR spectra (0-12 ppm region) of the dPG-HPA conjugates in $\mathrm{D}_{2} \mathrm{O}$ with varying phenolic substitutions.

Fig. 4a shows that all the dPG based crosslinking caused a higher nanogel yield compared to the linear PEG analogs, even for $\mathrm{dPG}-2 \%$ HPA, which suggests a preferable enzyme access and hence conversion rate towards the dendritic architecture. The slight increase in the phenolic substitution on dPG from $2 \%$ to $4 \%$ led to a higher nanogel yield. It was surprising that higher yield was not observable from the nanogels crosslinked with dPG-6\% HPA and dPG-8\% HPA, which means that some residual phenol groups must have remained unreacted in the polymers. The reason might be that further enzymatic crosslinking was not feasible when protein and substrates (dPG-HPA) were tightly restricted in gel networks to the extent that enzymes could not access substrates anymore.

Apart from the degree of phenolic substitution, $\mathrm{H}_{2} \mathrm{O}_{2}$ concentration is another important parameter that can be used to tune the crosslinking for a better nanogel yield. It is found that crosslinking conversion was notably enhanced at elevated $\mathrm{H}_{2} \mathrm{O}_{2}$ levels for PEG and dPG-2\% HPA, while there was no significant influence upon dPG loaded higher than $2 \%$ HPA. This result can be also explained by the full restriction of enzymes in the highly crosslinked gel networks. Nevertheless, the obtained results disclosed that more than $4 \%$ phenolic loading and higher $\mathrm{H}_{2} \mathrm{O}_{2}$ concentration were beneficial for the nanogel yield.

However, some earlier reports revealed that HRP activity could be irreversibly inactivated in the presence of a high concentration of phenols and $\mathrm{H}_{2} \mathrm{O}_{2}$ due to the substrate inhibition. ${ }^{28}$ The observation was fully supported by our findings for HRP's quick deactivation under the highly concentrated $\mathrm{H}_{2} \mathrm{O}_{2}$ conditions (Fig. 4b). HRP lost over 95\% activity after 24 hour incubation with $>22 \mathrm{mM} \mathrm{H}_{2} \mathrm{O}_{2}$ conditions but at low $\mathrm{H}_{2} \mathrm{O}_{2}$ level, e.g., less than $40 \mu \mathrm{M}$, no HRP deactivation, however, could be observed. This finding demonstrates that the control of initial and residual content of $\mathrm{H}_{2} \mathrm{O}_{2}$ for nanogel preparation is essential for obtaining optimal HRP activity. Besides the investigation of $\mathrm{H}_{2} \mathrm{O}_{2}$ influence, HRP stability was further studied with the incubation conditions of $100 \mathrm{mg} \mathrm{mL}^{-1} \mathrm{dPG}$ loaded with $0,2,4,6$, and $8 \%$ phenol groups, respectively. 
(a)

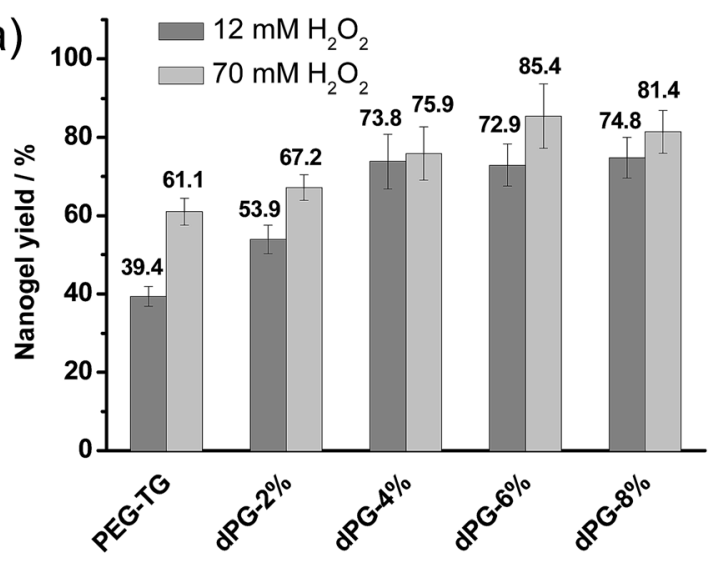

(c)

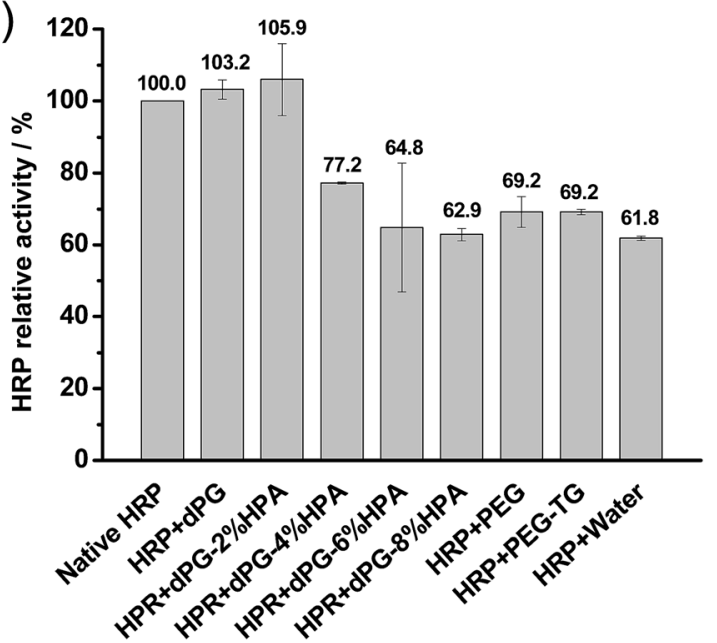

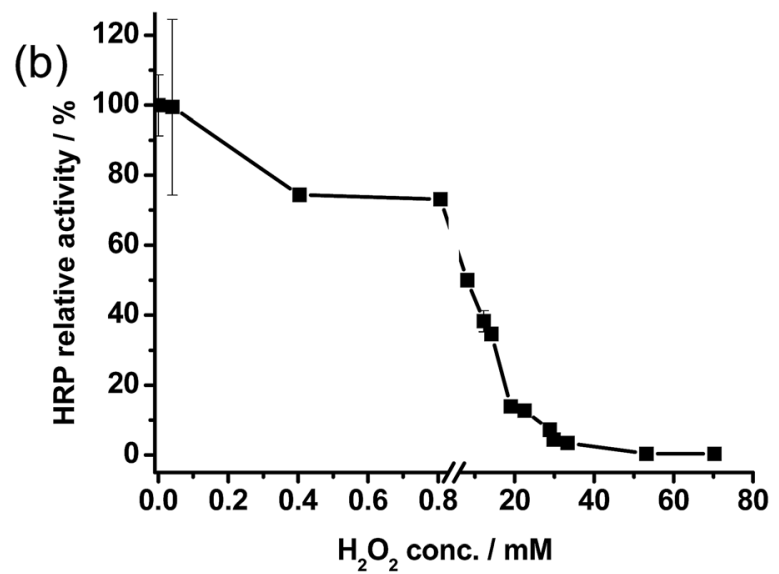

(d)

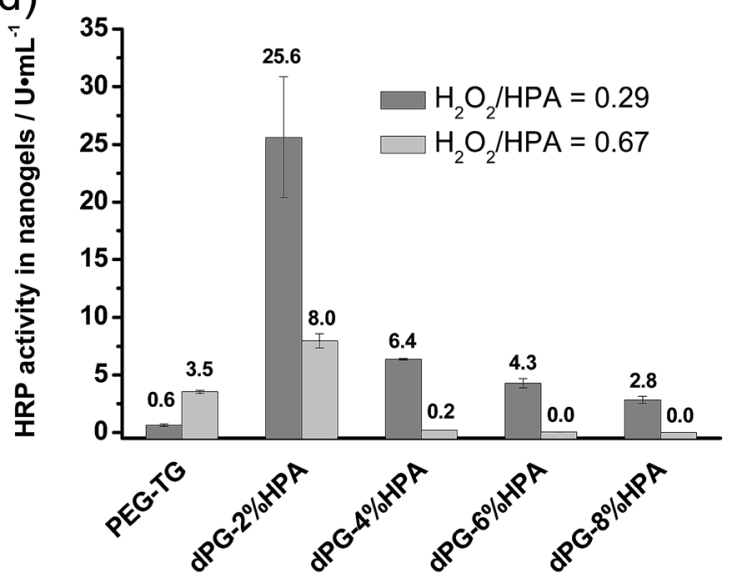

Fig. 4 Optimization of crosslinking conditions for HRP activity in dPG nanogels: (a) the influence of phenolic functionality on dPG to nanogel yield, (b) HRP stability ( $24 \mathrm{~h}$ ) in $\mathrm{H}_{2} \mathrm{O}_{2}$ solutions while $100 \%$ was set for the activity measured directly from HRP stock solutions, (c) HRP stability (24 h) in polymer solutions while $100 \%$ was set for the activity measured directly from HRP stock solutions, and (d) optimized HRP activity in dPG nanogels where volumetric activity $\left(\mathrm{U} \mathrm{mL}^{-1}\right)$ was used to show the total catalytic performance of all nanogels in one $\mathrm{mL}$.

Interestingly, it was observed that the addition of dPG alone or only loading $2 \%$ phenol groups helped stabilize HRP with $100 \%$ activity for at least 24 hours at room temperature, as is shown in Fig. 4c. This stabilization effect illustrates dPG's excellent biocompatibility as well as its high potential as additives or carriers for applications in protein technology. But further increasing of the phenol group loading on dPG impaired the dPG biocompatibility; decreased HRP activity was observed for the incubation with dPG-4\%-, 6\%- and 8\% HPA. This is in accordance with an early observation in the literature that reported HRP deactivation in a highly concentrated phenol solutions. $^{29}$

Based on the data in Fig. 4a-c, nanogel formulation for HRP activity should consider both the crosslinking yield and HRP's stability. Thus dPG nanogels were prepared with varied concentrations of $\mathrm{H}_{2} \mathrm{O}_{2}$ and phenolic loading of polymers while fixing the molar ratio of $\mathrm{H}_{2} \mathrm{O}_{2}$ / HPA at 0.29 and 0.67 , respectively, to assure full consumption of $\mathrm{H}_{2} \mathrm{O}_{2}$ in the end. Fig. 4d shows that there was always higher HRP activity in nanogels prepared at low phenolic loading polymers and $\mathrm{H}_{2} \mathrm{O}_{2}$ level. This finding revealed that HRP stability is more important than the protein loading efficiency in the pursuit for high HRP activity in dPG nanogels. The nanogels with the highest HRP activity were formulated at dPG-2\% HPA with $\mathrm{H}_{2} \mathrm{O}_{2} / \mathrm{HPA}$ at 0.29 , where the substrate inhibition was least. This data further demonstrated that HRP activity in nanogels greatly decreased if the $\mathrm{H}_{2} \mathrm{O}_{2}$ level was elevated from $\mathrm{H}_{2} \mathrm{O}_{2} / \mathrm{HPA}$ at 0.29 to 0.67 during preparation. Interestingly, dPG-2\% HPA nanogels had higher HRP activity than PEG-based nanogels, suggesting that protein loading efficiency took effect when the level of $\mathrm{H}_{2} \mathrm{O}_{2}$ and phenol groups was equal. These data helped us to conclude that the best nanogel activity could be accomplished by enzymatic crosslinking of dPG-2\% HPA at lower $\mathrm{H}_{2} \mathrm{O}_{2}$ level.

\section{Encapsulation of the second enzyme CalB in dPG nanogels}

In order to exploit the universality of the currently investigated method for protein encapsulation, a second enzyme, Candida antarctica lipase B (CalB), which is not involved in crosslinking 
(a)

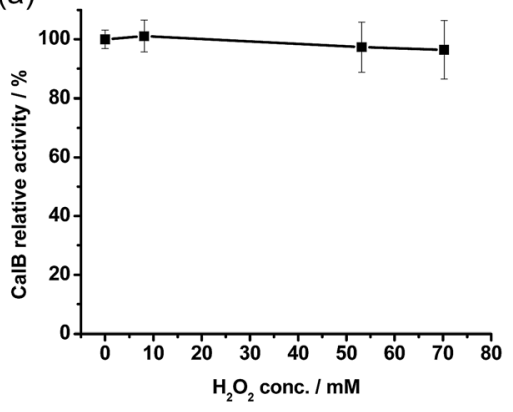

(b)

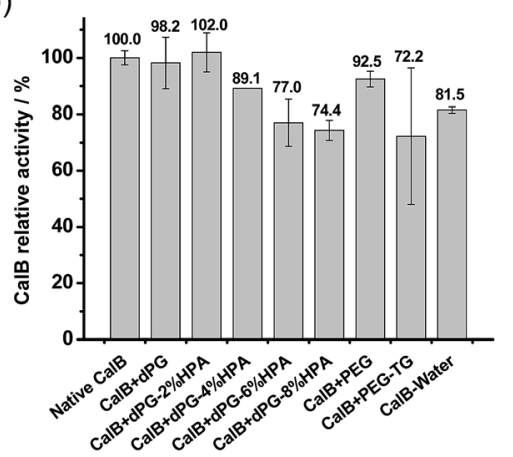

(c)

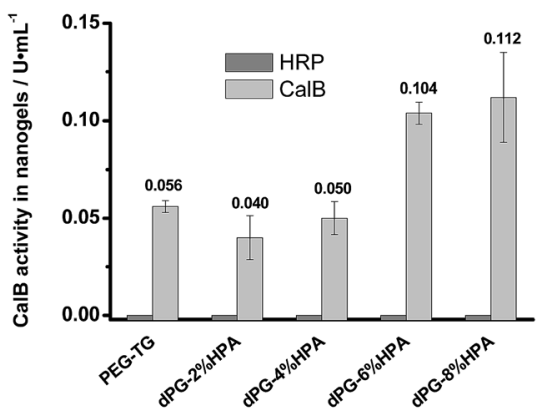

Fig. 5 Optimization of crosslinking conditions for CalB activity in dPG nanogels: (a) CalB stability (overnight) in $\mathrm{H}_{2} \mathrm{O}_{2}$ solutions while $100 \%$ was set for the activity measured directly from CalB stock solutions, (b) CalB stability (overnight) in polymer solutions while $100 \%$ was set for the activity measured directly from CalB stock solutions, and (c) optimized CalB activity in $\mathrm{dPG}$ nanogels where volumetric activity (U mL $\mathrm{mL}^{-1}$ ) was used to show the total catalytic performance of all nanogels in one $\mathrm{mL}$.

reactions, was also added during nanogel preparation. DLS data (Fig. S19, ESI $\dagger$ ) displayed that addition of the second enzyme to nanogels did not change the particle size. Furthermore, pronounced CalB activity could be detected in the nanogels (Experimental section), which confirmed that the "enzymatic nanogelation" approach was versatile for the encapsulation of different enzymes.

Similar to HRP encapsulation, CalB activity in nanogels was also needed to be optimized. But unlike HRP, CalB is a robust enzyme against many aggressive operation conditions, thus an associated inhibition by $\mathrm{H}_{2} \mathrm{O}_{2}$ and phenol groups might not be expected. To test this hypothesis, native CalB was incubated with $\mathrm{H}_{2} \mathrm{O}_{2}$ solutions with a large concentration gradient. The data in Fig. 5a revealed that CalB maintained nearly $100 \%$ activity after overnight incubation at $72 \mathrm{mM}$ concentration of $\mathrm{H}_{2} \mathrm{O}_{2}$, a level far exceeding the amount needed for nanogel preparation. Based on this finding, it can be concluded that the encapsulation of CalB can be operated even at higher $\mathrm{H}_{2} \mathrm{O}_{2}$ level.

Moreover, CalB stability was investigated by incubating the enzymes in solutions of $100 \mathrm{mg} \mathrm{mL}^{-1} \mathrm{dPG}$, phenolic derivatized dPG, PEG, and PEG-TG, respectively. Although deactivation of CalB by the incubation of polymer solutions was also found (Fig. 5b), the stability of CalB was notably enhanced beyond that of HRP with the same incubation conditions. The better CalB stability has profited from its intrinsic stability, while the overloaded phenolic moieties on the dPG levelled off dPG's biocompatibility, thus decreasing CalB activity accordingly. Therefore, CalB activity of dPG nanogels could be optimized by tuning both the crosslinking yield and dPG's phenolic functionality degree.

As what mentioned above, the best HRP activity of nanogels could be obtained under the crosslinking conditions with a low $\mathrm{H}_{2} \mathrm{O}_{2}$ concentration and less functionalized dPG. This condition will be particularly favourable for encapsulating a second enzyme, e.g., glucose oxidase, for coupled enzymatic reactions in biochemical assays. ${ }^{30,31}$ However, in some cases, the active version of HRP in nanogels is unwanted; for example, it may compete with CalB for substrates during formation of peroxycarboxylic acids in the presence of $\mathrm{H}_{2} \mathrm{O}_{2} \cdot{ }^{32,33}$ Consequently, in order to demonstrate the possibility to fully suppress HRP activity nanogels were fabricated with $72 \mathrm{mM} \mathrm{H}_{2} \mathrm{O}_{2}$ for encapsulation with two enzymes. Surprisingly, the nanogels were obtained at such high level of $\mathrm{H}_{2} \mathrm{O}_{2}$ concentrations, as seen in Fig. S20 (see the ESI $\dagger$ ); the separation was conducted without using any quenching steps. This indicated that all HRP was inactivated after crosslinking due to the strong $\mathrm{H}_{2} \mathrm{O}_{2}$ inhibition. Further activity assays of both CalB and HRP in nanogels confirmed the HRP's deactivation, and disclosed a prominent CalB activity, as shown in Fig. $5 \mathrm{c}$. The highest CalB activity was reached when $8 \%$ of phenol groups on $\mathrm{dPG}$ were used for the nanogel preparation, which suggested that the nanogel yield was a predominant factor in the increased CalB activity for preparation. This is also in agreement with previous findings of better CalB stability in regard to the influence from dPG-HPA and $\mathrm{H}_{2} \mathrm{O}_{2}$ than HRP's stability.

\section{Conclusions}

In summary, spherical dPG nanogels have been successfully fabricated by HRP-catalyzed oxidative crosslinking with an inverse miniemulsion template. The preparation method, which can be performed under mild physiological conditions, is particularly beneficial for bio-related delivery systems including drug and/or protein delivery. The success of this method opens up new horizons in nanogel research as it provides controlled crosslinking conditions so that quenching steps can be avoided, thus making it applicable for other polymeric nanogel preparation approaches.

Moreover, the encapsulation provides a robust and biocatalytically active nano-architecture that can withstand high temperatures and continuous operation, which suggests that it might be worthy of being tried for applications in other adverse conditions like organic solvents, extreme $\mathrm{pH}$ and high ionic strength. In addition, the successful loading of CalB into the carriers indicates the high potential of "enzymatic nanogelation" for encapsulation of diverse enzymes, in particular for applications related to coupled-enzyme reactions. 
Last but not least, HRP and CalB activity in nanogels can be optimized, depending on the preparation conditions. Conceivably, the system can be further improved, for example, by employing a wide range of two parameters $\left(\mathrm{H}_{2} \mathrm{O}_{2}\right.$ concentration, and polymer functionality), which should be of use for rational enzyme activity.

\section{Experimental section}

\section{Materials}

In general, chemicals were purchased from Sigma-Aldrich and used as received. Anhydrous pyridine and dimethylformamide (DMF) were purchased from Acros Organics. Deuterated solvents were bought from Deutero GmbH. dPG (Mw 6 kDa) was synthesized by anionic, ring-opening multi-branching polymerization of glycidol under slow monomer addition. ${ }^{34,35}$ Polyethylene glycol (PEG, Mw $6 \mathrm{kDa}$ ) was obtained from Acros Organics. Horseradish peroxidase (HRP, type VI-A, lyophilized powder) was purchased from Sigma-Aldrich. Candida antarctica lipase B (CalB, Lipozyme CALB L, protein content $0.24 \% \mathrm{w} / \mathrm{w}$ ) was a generous gift from Novozymes and was further purified through dialysis in water and lyophilized to powder before use.

dPG-HPA loaded with 2, 4, 6, and 8\% phenol groups was synthesized in five steps according to our previously reported procedure. ${ }^{21}$ Typically, dPG was initially converted to dPG-NH in three steps: mesylation, nucleophilic substitution, reduction (see the ESI $\dagger$ ). Subsequently, HPA was conjugated to dPG-NH through amide coupling reactions. The final compound dPG-HPA was purified through dialysis (membrane Mw cut off $1.5 \mathrm{kDa}$ ) again methanol for three days (see synthetic details and characterization in the ESI $\dagger$ ).

Sonicator $^{\mathrm{TM}}$ (Model W-220f, from Heat Systems-Ultrasonics, Inc.) was employed to emulsify aqueous solutions in cyclohexane as nano-sized droplets. Dynamic light scattering (DLS) measurements were carried out using a Zetasizer Nano-ZS from Malvern Instruments equipped with a $633 \mathrm{~nm} \mathrm{He}-\mathrm{Ne}$ laser. Nanogels were visualized through transmission electron microscopy (TEM, a Philips CM12 electron microscope) with cryo-TEM techniques. ${ }^{1} \mathrm{H}$ NMR spectra $(500 \mathrm{MHz})$ were recorded on a Delta JEOL Eclipse at $25{ }^{\circ} \mathrm{C}$ using $\mathrm{D}_{2} \mathrm{O}, \mathrm{CD}_{3} \mathrm{OD}$, and $\mathrm{CDCl}_{3}$ as solvents. The spectra were calibrated using the solvent residual peak. FTIR and UV-Vis were carried out on Jasco FT/IR4100 and Perkin-Elmer Lambda2S, respectively.

\section{Fabrication of dPG nanogels}

An inverse miniemulsion was created for making nanogels according to the modified procedure. ${ }^{13}$ Generally, a $190 \mu \mathrm{L}$ mixture of dPG-HPA and HRP solutions was pre-emulsified by magnetic stirring for $20 \mathrm{~min}$ in $5 \mathrm{~mL}$ cyclohexane containing $20 \mathrm{mg} \mathrm{mL}{ }^{-1}$ Span 80 and $67 \mathrm{mg} \mathrm{mL}^{-1}$ Tween 80 . The mixture under ice bath was completely emulsified by a tip sonicator for $4 \mathrm{~min}$, and during the course, $60 \mu \mathrm{L}$ volume of $\mathrm{H}_{2} \mathrm{O}_{2}$ solutions was introduced to initiate the enzymatic crosslinking. The amount of dPG-HPA and HRP used was excessive with final concentrations in aqueous phase at $100 \mathrm{mg} \mathrm{mL}^{-1}$ and $0.25 \mathrm{mg} \mathrm{mL} \mathrm{mL}^{-1}$, respectively; the $\mathrm{H}_{2} \mathrm{O}_{2}$ level was varied on demand. A sufficient period of time, e.g., more than two hours, was given to allow the enzymatic crosslinking to complete, which, on the other hand, fully consumed $\mathrm{H}_{2} \mathrm{O}_{2}$.

In order to study the possibility of encapsulating a second enzyme, a mixture was prepared with $100 \mathrm{mg} \mathrm{mL}^{-1}$ polymer and $1 \mathrm{mg} \mathrm{mL}{ }^{-1} \mathrm{HRP}$ and $0.6 \mathrm{mg} \mathrm{mL}^{-1}$ CalB. The mixture was then emulsified and crosslinked as nanogels with the above mentioned procedure.

hPG nanogels were purified first by centrifuging the miniemulsion at $10000 \mathrm{rpm}$ for 30 minutes before removing the supernatant. The pellet was further washed 3 times with $15 \mathrm{~mL}$ cyclohexane to remove surfactants. Subsequently, residual cyclohexane was removed by washing it 3 times in methanol (totally $15 \mathrm{~mL}$ ). Finally, pure nanogels were obtained by washing twice with water and then diluted up to $10 \mathrm{mg} \mathrm{mL}^{-1}$ as stock solutions for characterization. The yield was quantified in regard to the nanogel net weight to a totally used up mass after lyophilizing the nanogels.

\section{Assessment of the catalytic performance of free and encapsulated HRP}

The HRP activity was determined by the conversion of chromogenic substrate of ABTS (2,2'-azino-bis(3-ethylbenzothiazoline-6-sulfonic acid)). The obtained nanogels in each preparation batch were concentrated into one $\mathrm{mL}$ for activity determination. Typically, a $1.4 \mathrm{~mL}$ mixture of substrate solutions was prepared to contain $2.5 \mathrm{mM}$ ABTS and $0.26 \mathrm{mM} \mathrm{H}_{2} \mathrm{O}_{2}$ in $100 \mathrm{mM}$ phosphate buffer ( $\mathrm{pH}$ 7). Subsequently, $50 \mu \mathrm{L}$ droplets of aqueous solutions of native HRP or nanogels were added into the mixture to initiate the reaction at room temperature. The absorbance increase was recorded at $436 \mathrm{~nm}$ by UV-Vis to determine an initial reaction rate period within 2 min. All the reactions were performed in triplicate. In order to simplify the calculation, HRP activity (U) was defined as the absorbance increase per min of free HRP or nanogels. As indicated in the literature, ${ }^{36,37}$ a relative activity (\%) was always used to show the tendency of activity changes during enzyme stability and reuse studies, where $100 \%$ was set for the activity measured directly from HRP stock solutions. Volumetric activity $\left(\mathrm{U} \mathrm{mL}^{-1}\right)$ was calculated to reflect the total catalytic performance of nanogels.

\section{Assessment of the catalytic performance of free and encapsulated CalB}

Prior to the catalytic assessment, the nanogels prepared during optimization were concentrated into one $\mathrm{mL}$ as stock solution for use. Determination of the CalB activity was conducted by hydrolysis of 4 -nitrophenyl propionate $\left(p\right.$-NPB).$^{38}$ In general, $50 \mu \mathrm{L}$ enzyme solutions (free CalB or nanogels) were added to $1.45 \mathrm{~mL}$ substrate solutions which contained $2 \mathrm{mM} p$-NPB in $40 \%$ (wt/wt) ethanol solutions at room temperature. The absorbance increase was recorded at $410 \mathrm{~nm}$ to obtain the initial reaction rate period within $5 \mathrm{~min}$. All reactions were repeated in triplicate. Similar to HRP activity definition, CalB activity (U) was expressed as the absorbance increase per min of the free or encapsulated enzymes. Again, the relative activity (\%) 
was employed for the CalB stability study, and volumetric activity $\left(\mathrm{U} \mathrm{mL}^{-1}\right)$ was used for the expression of total catalytic performance of nanogels.

\section{Cryogenic transmission electron microscopy}

A droplet $(5 \mu \mathrm{L})$ of the sample solutions was placed on hydrophilized (plasma treatment using a BALTEC MED 020 device (Leica Microsystems, Wetzlar, Germany)), perforated carbon filmed grids (Quantifoil Micro Tools GmbH, Jena, Germany). The excess fluid was blotted off to create an ultra-thin layer (typical thickness of 200-300 nm) of the solutions, which spans the holes of the support film. The prepared samples were immediately vitrified by propelling the grids into liquid ethane at its freezing point $\left(-184^{\circ} \mathrm{C}\right)$. The vitrified sample grids were transferred under liquid nitrogen by the use of a Gatan (Pleasanton, CA, USA) cryo-holder (Model 626) into a Tecnai F20 TEM (FEI company, Oregon, USA) equipped with FEG and operated at $160 \mathrm{kV}$ acceleration voltage. Microscopy was carried out at $-175{ }^{\circ} \mathrm{C}$ sample temperature using the microscope's low dose protocol at calibrated primary magnifications of $29 \mathrm{k}$. The defocus was set to be $9.81 \mu \mathrm{m}$. Images were recorded by the use of a $4 \mathrm{k}$-Eagle CCD camera (FEI Company, Oregon, USA) at $2 \mathrm{k}$ resolutions (binning factor 2).

\section{Acknowledgements}

We thank the BMBF IGCST project, the DFG (Core-Facility BioSupraMol) and the Cluster of Excellence "Unifying Concepts in Catalysis" (UniCat) for financial support. We are grateful to Dr Florian Paulus (FU Berlin) for providing hyperbranched polyglycerol and to Dr Pamela Winchester (FU Berlin) for proofreading this manuscript.

\section{Notes and references}

1 A. V. Kabanov and S. V. Vinogradov, Angew. Chem., Int. Ed., 2009, 48, 5418-5429.

2 W. Chen, F. Meng, J. Feijen and Z. Zhong, J. Controlled Release, 2013, 172, e115.

3 S. Singh, F. Topuz, K. Hahn, K. Albrecht and J. Groll, Angew. Chem., Int. Ed., 2013, 52, 3000-3003.

4 K. Malzahn, W. D. Jamieson, M. Droge, V. Mailander, A. T. A. Jenkins, C. K. Weiss and K. Landfester, J. Mater. Chem. B, 2014, 2, 2175-2183.

5 H.-s. Peng, J. A. Stolwijk, L.-N. Sun, J. Wegener and O. S. Wolfbeis, Angew. Chem., Int. Ed., 2010, 49, 4246-4249.

6 Y. Sasaki and K. Akiyoshi, Chem. Rec., 2010, 10, 366-376.

7 J. Jing, D. Alaimo, E. De Vlieghere, C. Jerome, O. De Wever, B. G. De Geest and R. Auzely-Velty, J. Mater. Chem. B, 2013, 1, 3883-3887.

8 S. Yu, P. Yao, M. Jiang and G. Zhang, Biopolymers, 2006, 83, 148-158.

9 K. Kim, B. Bae, Y. J. Kang, J.-M. Nam, S. Kang and J.-H. Ryu, Biomacromolecules, 2013, 14, 3515-3522.
10 K. Akiyoshi, S. Kobayashi, S. Shichibe, D. Mix, M. Baudys, S. Wan Kim and J. Sunamoto, J. Controlled Release, 1998, 54, 313-320.

11 D. Steinhilber, A. L. Sisson, D. Mangoldt, P. Welker, K. Licha and R. Haag, Adv. Funct. Mater., 2010, 20, 4133-4138.

12 D. A. Heller, Y. Levi, J. M. Pelet, J. C. Doloff, J. Wallas, G. W. Pratt, S. Jiang, G. Sahay, A. Schroeder, J. E. Schroeder, Y. Chyan, C. Zurenko, W. Querbes, M. Manzano, D. S. Kohane, R. Langer and D. G. Anderson, Adv. Mater., 2013, 25, 1449-1454.

13 D. Steinhilber, S. Seiffert, J. A. Heyman, F. Paulus, D. A. Weitz and R. Haag, Biomaterials, 2011, 32, 1311-1316.

14 S. E. A. Gratton, P. D. Pohlhaus, J. Lee, J. Guo, M. J. Cho and J. M. DeSimone, J. Controlled Release, 2007, 121, 10-18.

15 L. S. Moreira Teixeira, J. Feijen, C. A. van Blitterswijk, P. J. Dijkstra and M. Karperien, Biomaterials, 2012, 33, 1281-1290.

16 D. J. Menzies, A. Cameron, T. Munro, E. Wolvetang, L. Grøndahl and J. J. Cooper-White, Biomacromolecules, 2012, 14, 413-423.

17 L.-S. Wang, C. Du, W. S. Toh, A. C. A. Wan, S. J. Gao and M. Kurisawa, Biomaterials, 2014, 35, 2207-2217.

18 R. Jin, L. S. M. Teixeira, P. J. Dijkstra, Z. Zhong, C. A. v. Blitterswijk, M. Karperien and J. Feijen, Tissue Eng., Part A, 2010, 16, 2429-2440.

19 B. Y. Kim, J. W. Bae and K. D. Park, J. Controlled Release, 2013, 172, 535-540.

20 K. M. Park, Y. Lee, J. Y. Son, D. H. Oh, J. S. Lee and K. D. Park, Biomacromolecules, 2012, 13, 604-611.

21 C. Wu, C. Strehmel, K. Achazi, L. Chiappisi, J. Dernedde, M. C. Lensen, M. Gradzielski, M. B. Ansorge-Schumacher and R. Haag, Biomacromolecules, 2014, 15, 3881-3890.

22 S. Kobayashi, H. Uyama and S. Kimura, Chem. Rev., 2001, 101, 3793-3818.

$23 \mathrm{X} . \mathrm{Xu}, \mathrm{A}$. K. Jha, D. A. Harrington, M. C. Farach-Carson and X. Jia, Soft Matter, 2012, 8, 3280-3294.

24 W. Wu, S. Chen, Y. Hu and S. Zhou, J. Diabetes Sci. Technol., 2012, 6, 892-901.

25 A. Dan, C. Kotsmar, J. K. Ferri, A. Javadi, M. Karbaschi, J. Kragel, R. Wustneck and R. Miller, Soft Matter, 2012, 8, 6057-6065.

26 M. N.-I. Walters, J. M. Papadimitriou, T. A. Robertson and J. M. Archer, J. Pathol., 1970, 100, 31-37.

27 A. Prokop, E. Kozlov, G. W. Newman and M. J. Newman, Biotechnol. Bioeng., 2002, 78, 459-466.

28 K. J. Baynton, J. K. Bewtra, N. Biswas and K. E. Taylor, Biochim. Biophys. Acta, Protein Struct. Mol. Enzymol., 1994, 1206, 272-278.

29 K. J. Baynton, J. K. Bewtra, N. Biswas and K. E. Taylor, Biochim. Biophys. Acta, Protein Struct. Mol. Enzymol., 1994, 1206, 272-278.

30 W. M. Aumiller, B. W. Davis, N. Hashemian, C. Maranas, A. Armaou and C. D. Keating, J. Phys. Chem. B, 2014, 118, 2506-2517.

31 F. Costantini, R. Tiggelaar, S. Sennato, F. Mura, S. Schlautmann, F. Bordi, H. Gardeniersb and C. Manetti, Analyst, 2013, 138, 5019-5024. 
32 F. Björkling, S. E. Godtfredsen and O. Kirk, J. Chem. Soc., Chem. Commun., 1990, 1972-1995.

33 S. Warwel and M. R. g. Klaas, J. Mol. Catal. B: Enzym., 1995, 1, 29-35.

34 A. Sunder, R. Mülhaupt, R. Haag and H. Frey, Adv. Mater., 2000, 12, 235-239.

35 A. Sunder, R. Hanselmann, H. Frey and R. Mülhaupt, Macromolecules, 1999, 32, 4240-4246.
36 J. Ratzka, L. Lauterbach, O. Lenz and M. B. AnsorgeSchumacher, J. Mol. Catal. B: Enzym., 2012, 74, 219-223.

37 C. Wu, S. Bai, M. B. Ansorge-Schumacher and D. Wang, Adv. Mater., 2011, 23, 5694-5699.

38 D. Pérez, S. Martín, G. Fernández-Lorente, M. Filice, J. M. Guisán, A. Ventosa, M. T. García and E. Mellado, PLoS One, 2011, 6, e23325. 